CLINICAL STUDY

\title{
Chemerin is expressed mainly in pancreas and liver, is regulated by energy deprivation, and lacks day/night variation in humans
}

\author{
John P Chamberland ${ }^{1,2}$, Reena L Berman ${ }^{1,2}$, Konstantinos N Aronis ${ }^{1,2,3}$ and Christos S Mantzoros ${ }^{1,2,3}$ \\ ${ }^{1}$ Section of Endocrinology, Boston VA Healthcare System, Harvard Medical School, Boston, Massachusetts O2130, USA, ${ }^{2}$ Division of Endocrinology, \\ Diabetes and Metabolism, Department of Medicine, Harvard Medical School, Beth Israel Deaconess Medical Center, 330 Brookline Avenue, Feldberg 876 , \\ Boston, Massachusetts 02215, USA and ${ }^{3}$ Department of Medicine, Boston Medical Center, Boston University, Boston, Massachusetts O2118, USA \\ (Correspondence should be addressed to C S Mantzoros at Division of Endocrinology, Diabetes and Metabolism, Department of Medicine, \\ Beth Israel Deaconess Medical Center, Harvard Medical School; Email: cmantzor@bidmc.harvard.edu)
}

\begin{abstract}
Objective: Chemerin is an adipocyte-secreted hormone and has recently been associated with obesity and the metabolic syndrome. Although studies in rodents have outlined the aspects of chemerin's function and expression, its physiology and expression patterns are still to be elucidated in humans. Methods: To evaluate for any day/night variation in chemerin secretion, we analyzed hourly serum samples from six females in the fed state. To examine whether energy deprivation affects chemerin levels, and whether this could be mediated through leptin, we analyzed samples from the same subjects in the fasting state while administering either placebo or leptin. To evaluate for any potential doseeffect relationship between leptin and chemerin, we administered increasing metreleptin doses to five females. A tissue array was used to study the expression of chemerin in different human tissues. Ex vivo treatment of human fat explants from three subjects with leptin was carried out to evaluate for any direct effect of leptin on adipocyte chemerin secretion.

Results: Chemerin does not display a day/night variation, while acute energy deprivation resulted in a significant drop in circulating chemerin levels by $\sim 42 \%$. The latter was unaltered by metreleptin administration, and leptin administration did not affect the secretion of chemerin by human adipose tissue studied ex vivo. Chemerin was expressed primarily in the pancreas and liver. Chemerin receptor showed increased expression in the lymph nodes and the spleen.

Conclusions: We outline for the first time chemerin expression and physiology in humans, which are different from those in mice.
\end{abstract}

European Journal of Endocrinology 169 453-462

\section{Introduction}

With the emergence of obesity as a major health hazard, several adipose tissue-secreted proteins and hormones, known as adipokines, have been identified and linked to metabolic regulation. Changes in the secretion of many of these molecules are now thought to play a major role in the development of obesity and obesity-related diseases. Chemerin (RARRES2) is one such protein that has recently been proposed to be an adipokine. It is secreted as an inactive $18-\mathrm{kDa}$ pro-peptide and is subsequently cleaved to a $16-\mathrm{kDa}$ active protein that binds to the $\mathrm{G}$ protein-couple receptor, chemokine-like receptor 1 (CMKLR1) (1). Chemerin was first identified in psoriatic skin lesions (2) and is now known to be involved in the recruitment of immune cells such as immature dendritic cells, macrophages, natural killer cells, and monocytes to the sites of tissue inflammation or injury (3). Chemerin is associated with proinflammatory cytokines such as tumor necrosis factor $\alpha$ (TNF- $\alpha$ ) both in vitro and in vivo $(4,5)$. Recently, it has been demonstrated that chemerin promotes angiogenesis in endothelial cells $(6,7)$. A major role of chemerin is adipogenesis regulation and it has also been associated with the metabolic syndrome $(8,9,10)$. Chemerin has been shown to be correlated with BMI, homeostasis model assessment of insulin resistance, blood pressure, and circulating triglycerides $(10,11)$ in humans. Furthermore, increased chemerin levels correlate with other obesity-related markers such as leptin, resistin, and C-reactive protein as well as HbA1c $(12,13,14)$. In vitro experiments have demonstrated that chemerin is an important regulator of adipocyte differentiation and metabolism, as exhibited by the knockdown of chemerin causing an impaired pre-adipocyte differentiation into mature adipocytes as well as reduced expression of glucose and lipid metabolism genes $(8,15)$. However, little is known about the circulatory or expression patterns of chemerin in humans.

Although chemerin has been proposed to be an adipokine, the expression pattern of chemerin in human tissues has not yet been evaluated comparatively. 
Furthermore, the physiology of chemerin in humans remains to be fully elucidated. No studies to date have determined whether chemerin displays a day/night pattern of secretion and what the effect of acute and/or chronic energy deprivation on circulating chemerin levels in humans is. It is expected that circulating levels of chemerin, a factor associated with energy homeostasis, would respond appropriately to changes in energy intake. Finally, any potential interactions between chemerin and other molecules affected by energy intake, such as leptin, have not yet been studied. Leptin is a 167 amino acid adipokine (16) that has a well-established role in the neuroendocrine regulation of energy homeostasis $(17,18)$. Recent studies have demonstrated an association between leptin levels, obesity, and the metabolic syndrome $(17,18,19)$. During states of starvation, circulating levels decrease and are responsible for changes in the neuroendocrine axis (20). In mouse models, leptin has been shown to act on the hypothalamic pro-opiomelanocortin neurons to decrease food intake and maintain glucose homeostasis (21). Studies on patients with conditions characterized by chronic energy deprivation, such as anorexia nervosa and hypothalamic amenorrhea, have demonstrated that low leptin levels are causally related to energy deprivation-induced suppression of neuroendocrine axes, whereas leptin administration restores these energy deprivation-induced changes $(20,22,23,24)$.

We used herein a human mRNA tissue array to evaluate the expression pattern of chemerin and its receptor in human tissues. We then proceeded to assess whether chemerin has a day/night variation pattern in humans, to determine whether acute energy deprivation (for 3 days) alters chemerin levels, to investigate for potential association with changes in circulating TNF- $\alpha$ as measured by tumor necrosis factor receptor II (TNFRII) as well as leptin-mediated effects of energy deprivation in humans, and to examine the tissue expression of chemerin. To these ends, we first used frequently collected samples from six healthy female individuals studied while in the isocaloric fed state, after 3 days of fasting, and 3 days of fasting plus metreleptin administration; then we used samples collected from another five healthy female subjects while treated with low, physiological, and supraphysiological doses of leptin; and finally, we performed ex vivo experiments involving adipose tissue surgically removed from human subjects.

\section{Subjects and methods}

\section{Tissue mRNA expression of chemerin and CMKLR 1}

Chemerin and CMKLR1 mRNA expressions were measured using human-specific TaqMan Gene Expression
Assays (Assay ID: Chemerin, Hs00161209_g1; CMKLR1, Hs01081979_s1, Applied Biosystems) in 7500 Fast Real-Time PCR system using Standard realtime 7500 protocol. Data were analyzed using 7500 system software (Applied Biosystems) and relative quantification was done using $\Delta \Delta C$ t method with $\beta$-actin as the internal control. Analysis of tissue mRNA expression was done in duplicate with TissueScan Real-Time Human Major Tissue Panel from OriGene, Rockville, MD, USA, containing 48 tissue types.

\section{Study 1}

Day/night variation analysis and effect of acute energy deprivation To evaluate whether chemerin has a day/night variation pattern, we collected serum samples from six healthy, lean, eumenorrheic women (age $22.8 \pm 3.4$ years; BMI $21.7 \pm 2.2 \mathrm{~kg} / \mathrm{m}^{2}$ ) initially studied in the isocaloric fed state (25). All female subjects had a regular menstrual cycle and were not taking any medications including oral contraceptive pills. Study visits were standardized at the 6th to 11th day of their menstrual cycle. The study was approved by the Institutional Review Board at the Beth Israel Deaconess Medical Center (BIDMC) and all participants gave informed consent. In order to evaluate whether energy deprivation has any effect on chemerin levels and whether such a change could be mediated through leptin, we collected samples from the same subjects on two separate admissions, spaced at least 8 weeks apart, in which subjects were randomized to receive either placebo or leptin during complete fasting for 3 days. Admissions in each state were held in the General Clinical Research Center (GCRC) of BIDMC for 4 days with standardized diet, exercise, and light/dark intervals. Baseline blood samples were taken at $0800 \mathrm{~h}$ on day 1 of each visit before intervention commenced. On day 3 of all three visits, blood samples were drawn every hour starting at $0800 \mathrm{~h}$ for $24 \mathrm{~h}$. For the admission in the fed state, subjects were given an isocaloric diet with breakfast at $0800 \mathrm{~h}$, lunch at $1300 \mathrm{~h}$, dinner at $1800 \mathrm{~h}$, and a snack at $2200 \mathrm{~h}$. The caloric intake was distributed so that $20 \%$ of calories were from breakfast, 35\% from lunch, 35\% from dinner, and $10 \%$ from the evening snack. For admissions in the fasting state, treatments with placebo or metreleptin were performed in a double-blinded, randomized, cross-over fashion. Replacement doses of metreleptin were given as four s.c. injections per day every $6 \mathrm{~h}$ starting at $0800 \mathrm{~h}$ on day 1 . Metreleptin was administered at doses of $0.08 \mathrm{mg} / \mathrm{kg}$ per day on day 1 and $0.2 \mathrm{mg} / \mathrm{kg}$ per day on days 2 and 3. Placebo treatment was carried out with the same schedule as well as the same volume and mode of administration as during leptin treatment. 


\section{Study 2}

Effect of increasing doses of leptin on chemerin circulation To evaluate for any potential dose-effect relationship of metreleptin administration on chemerin levels, we evaluated five healthy female volunteers in a cross-over study of administration of low $(0.01 \mathrm{mg} / \mathrm{kg})$, physiological $(0.1 \mathrm{mg} / \mathrm{kg})$, and high $(0.3 \mathrm{mg} / \mathrm{kg})$ metreleptin doses on three separate clinical visits (26). The mean age and the BMI of the subjects were 20.4 \pm 0.7 years and $21.9 \pm 0.7 \mathrm{~kg} / \mathrm{m}^{2}$ respectively. All female subjects who were in the isocaloric fed state had a regular menstrual cycle and were not taking any medications including oral contraceptive pills. The study was approved by the Institutional Review Board at the BIDMC and all participants gave informed consent. One-day admissions in each state were held in the GCRC of BIDMC with samples taken at 0800, 1200, 1600, and $2000 \mathrm{~h}$. Leptin at all doses was administered once at $0800 \mathrm{~h}$.

\section{Ex vivo treatment of adipose tissue}

Ex vivo procedures were carried out as previously reported (27). In short, human subcutaneous and omental fat explants obtained from three subjects (two females, one male, age $51.7 \pm 9.5$ years, BMI $43.8 \pm$ $5.8 \mathrm{~kg} / \mathrm{m}^{2}$ ) were placed in Krebs-Ringer-HEPES buffer (20 mmol/l, pH 7.4) containing 2.5\% BSA and $200 \mathrm{nmol} / \mathrm{l}$ adenosine in the operating room and were immediately taken to the laboratory. Tissue was then minced and all nonadipose tissue was removed by rinsing in fresh buffer. The samples were divided equally and incubated at $37^{\circ} \mathrm{C}$ in a shaking water bath with or without leptin treatment $(100 \mathrm{ng} / \mathrm{ml})$ for $20 \mathrm{~h}$. Chemerin secretion was measured via ELISA of the supernatant. Subcutaneous adipose tissue protein levels were measured by western blot analysis.

\section{Western blotting}

Western blotting was done as previously described (27). In short, the proteins were loaded on each lane and underwent SDS-PAGE. Proteins were then blotted onto nitrocellulose membranes (Schleicher \& Schuell, Inc., Keene, NH, USA). The membranes were blocked for $20 \mathrm{~min}$ in TBS containing 5\% nonfat dry milk and $0.1 \%$ Tween-20. Primary antibodies were incubated in TBS containing 5\% nonfat dry milk overnight and then incubated with HRP secondary antibodies for $2 \mathrm{~h}$. After incubation with all antibodies, membranes were washed with TBS containing 0.1\% Tween-20. Enhanced chemiluminescence was used for detection. Measurement of signal intensity on nitrocellulose membranes was performed using Image J (National Institutes of Health, Bethesda, MD, USA) processing and analysis software.

\section{Hormone measurements}

Serum chemerin was measured by ELISA (Biovendor, Chandler, NC, USA) with a sensitivity of $0.1 \mathrm{ng} / \mathrm{ml}$ and intra-assay $\mathrm{CV}$ of $5.1-7.0 \%$ and inter-assay $\mathrm{CV}$ of $6.9-8.3 \%(28,29)$. Serum soluble TNFRII (sTNFRII) was measured by ELISA (R\&D Systems, Minneapolis, MN, USA) with a sensitivity of $0.6 \mathrm{pg} / \mathrm{ml}$, intra-assay CV of $2.6-4.8 \%$ and inter-assay CV of $3.5-5.1 \%$. Serum leptin, insulin, IGF1, and free fatty acids (FFA) were measured as previously reported (25).

\section{Statistical analysis}

Statistical analysis was performed with STATA v11.2 (STATA Corp., College Station, TX, USA) and Pulse XP (University of Virginia, Charlottesville, VA, USA) software. Results are presented as means \pm s.D. Normality of the dependent variables was evaluated with the Shapiro-Wilkes test of normality and P-P plots. Evaluation of the existence of a day/night variation pattern for chemerin was performed by hour-to-hour comparison across a $24-\mathrm{h}$ period using repeated measurements ANOVA. Possible dominant harmonic components were analyzed on a subject-to-subject basis by spectral domain analysis for confirmation, using stata pergram routine. Chemerin, leptin, and sTNFRII levels were also analyzed for potential day/night variation by four parameters: cosine, nonlinear regression models, evaluating the adjusted coefficient of determination $\left(R^{2}\right)$. Comparisons of all molecules' levels between the three states (fed, fasting plus placebo, and fasting plus leptin) were performed with hierarchical mixed effects linear modeling. Chemerin levels were modeled as a linear function of time, at the level of each individual. Fixed effects of each state on the intercept and slope of the growth trajectories were assessed by introducing 'state' as a level-2 dummy variable. Comparisons between day 1 and day 4 and between and within each group as well as the study 2 leptin-dose response were performed by repeated measures ANOVA. Correlations between chemerin and insulin levels in the fasting state were evaluated with simple linear regression. Analysis of chemerin levels in $e x$ vivo experiment was done by paired $t$-test. All $P$ values are two-sided with the alpha criterion set to 0.05 .

\section{Results}

\section{Tissue mRNA expression of chemerin and CMKLR 1}

Values of chemerin and CMKLR1 mRNA expression levels are displayed as relative to adrenal gland levels. Chemerin levels are found distributed throughout multiple tissues with the highest expression seen in pancreas, liver, adrenal gland, kidney (Fig. 1), and rectum (data not shown). Chemerin is expressed the 

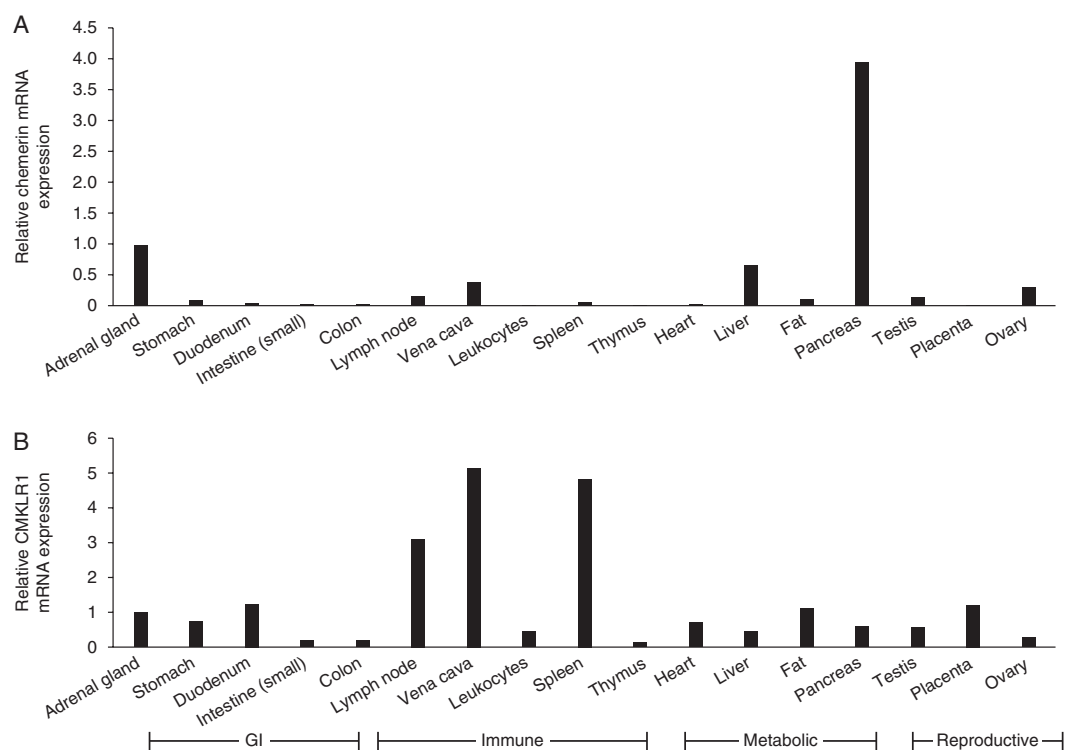

Figure 1 Tissue mRNA expression of chemerin and CMKLR1. Relative mRNA expression of chemerin (A) and CMKLR1 (B) in human tissues using human mRNA tissue array. Specific tissue type for each corresponding bar is labeled in the $x$-axis of each graph and a legend to group tissues by function in relation to both graphs $A$ and $B$ is located at the bottom of the figure. Methods of experiment are described in detail in the Subjects and methods section. least in the thymus, brain, plasma leukocytes, and placenta. The expression of CMKLR1 is more widely distributed while the highest levels are seen in tissues important in immune or inflammatory responses such as the spleen, lymph node, and vena cava (Fig. 1). Other tissues analyzed for chemerin and CMKLR1 include muscle, brain, intracranial nerves, retina, optic nerve, urethra, bladder, vagina, penis, uvula, oviduct, seminal vesicles, prostate, cervix, mammary gland, pituitary, thyroid, pericardium, tonsils, esophagus, nasal mucosa, trachea, tongue, spinal cord, epididymis, and skin (data not shown).

\section{Chemerin in circulation}

Baseline characteristics including weight, BMI, insulin, FFA, chemerin, serum sTNFRII, and leptin of the six subjects in all three conditions (fed, fasting plus placebo, and fasting plus metreleptin) are outlined in Table 1. Only the fasting plus metreleptin administration state demonstrated a slight decrease in body weight and BMI. Day 4 measurements of insulin, leptin, FFA, sTNFRII and chemerin were not statistically different from day 1 in the fed state. The level of insulin, leptin, and chemerin all decreased in response to fasting on day 4 compared with day 1 . In contrast, FFA increased in response to fasting. Soluble TNFRII was unchanged in all three states. Leptin was increased on day 4 in the fasting state with metreleptin administration while all other hormones remained in normal range seen with placebo administration in fasting condition (Table 1).

\section{Day/night variation of chemerin}

Repeated measurements ANOVA on normalized chemerin levels revealed no statistically significant difference between hourly time points $(P=0.169)$. The nonlinear cosinor regression analysis of chemerin over the 24-h period demonstrated a statistically significant day/night variation in both chemerin and sTNFRII circulating levels during the fed state (adjusted coefficients of determination: $R^{2}=8.45 \%, P<0.01$ and $R^{2}=4.8 \%, P=0.02$, respectively). Although statistically significant, we believe that the coefficient of determination calculated for chemerin and sTNFRII was not of a clinically significant magnitude. There are no available guidelines on what level of coefficient of determination, derived from non-linear regression models, should serve as a threshold to characterize a model as 'physiologically' or 'clinically' significant, and the statistical consensus recommends that this decision should be made on a study-to-study basis, based on the pre-existing literature. Cortisol and leptin are hormones with established day/night variability secretion patterns and thus using them as a reference is relevant. Previous studies from our group have demonstrated that cortisol yields an adjusted coefficient of determination of more than $30 \%$ in similar statistical models (30), while in this study leptin yielded an adjusted coefficient of determination of $27.66 \%(P<0.01)$. Given the fact that the coefficient of determination for hormones with well-established day/night variation patterns is in the $25-30 \%$ range, we support the notion that the $4.8-8.45 \%$ level observed in chemerin and sTNFRII circulating levels is not of clinical significance. Leptin levels displayed a significant day/night rhythm during the fed state with a peak during the night (Fig. 2).

\section{Effects of acute energy deprivation on chemerin}

Circulating chemerin levels were significantly lower after a 72-h fasting than during the fed state 


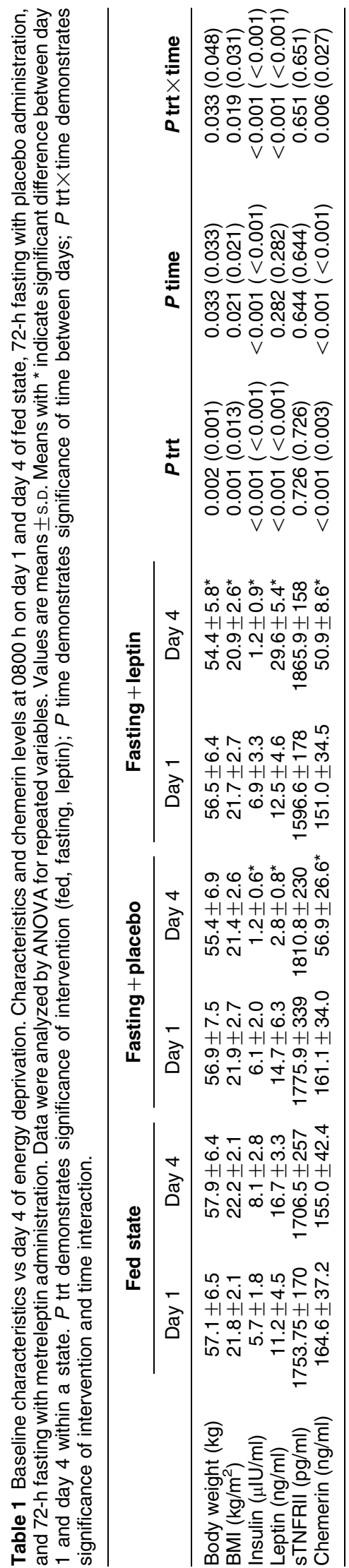

$(P<0.001)$. Similarly, leptin displayed decreased circulating levels in the fasting state compared with the fed state $(P<0.001)$. However, sTNFRII levels were not affected by a 72 -h fast $(P=0.557)$ (Fig. 3).

\section{Leptin effects on chemerin}

Leptin levels showed a significant drop during the fasting state compared with the fed state. Leptin replacement in the fasting state by metreleptin administration completely restored serum leptin levels to the physiological range (Table 1). On the contrary, chemerin levels were significantly lower in fasting state during metreleptin administration compared with during the fed state $(P<0.001)$. Also, levels of chemerin during leptin replacement were not statistically different from levels during fasting plus placebo $(P=0.84)$. Furthermore, levels of sTNFRII were unaffected by treatment with recombinant leptin $(P=0.715)$ (Fig. 3).

Leptin treatment at high, physiological, and low doses showed no difference in chemerin circulating levels (Fig. 4A). Similarly, treatment of subcutaneous and omental adipose tissue explants with leptin at $100 \mathrm{ng} / \mathrm{ml}$ for $20 \mathrm{~h}$ showed no effect on chemerin secretion and protein levels (Fig. 4B, C, and D).

\section{Correlations of chemerin with insulin in the fasting state}

Correlations of chemerin with insulin were also evaluated in the fasting state. In the beginning of the fasting period, the standardized regression coefficient between chemerin and insulin was $\beta=0.74$ $(P=0.11)$ while after 4 days of fasting it was $\beta=-0.7$ $(P=0.86)$. Regression of the change of insulin levels on chemerin levels also yielded a non-significant association $(\beta=0.24, P=0.35)$.

\section{Discussion}

Previous studies on mice have demonstrated that chemerin is a molecule almost exclusively expressed in adipose tissue and liver $(8,10)$. Furthermore, studies on humans have evaluated either the expression of chemerin in some tissues/cells (31) or in just adipose tissue (32), but a relative comparison of adipose tissue expression with all other tissue types has not been done. We thus comparatively evaluated for the first time chemerin expression in several human tissue types with that of adipose tissue, by the use of human tissue mRNA array. While liver still displays an increased relative expression of chemerin in humans, fat seems to express less chemerin when compared with other tissues such as pancreas and the adrenal gland. Furthermore, upon evaluation of the receptor of chemerin, CMKLR1, we show that it is almost evenly dispersed through multiple human tissues including fat, lung, the adrenal gland, 

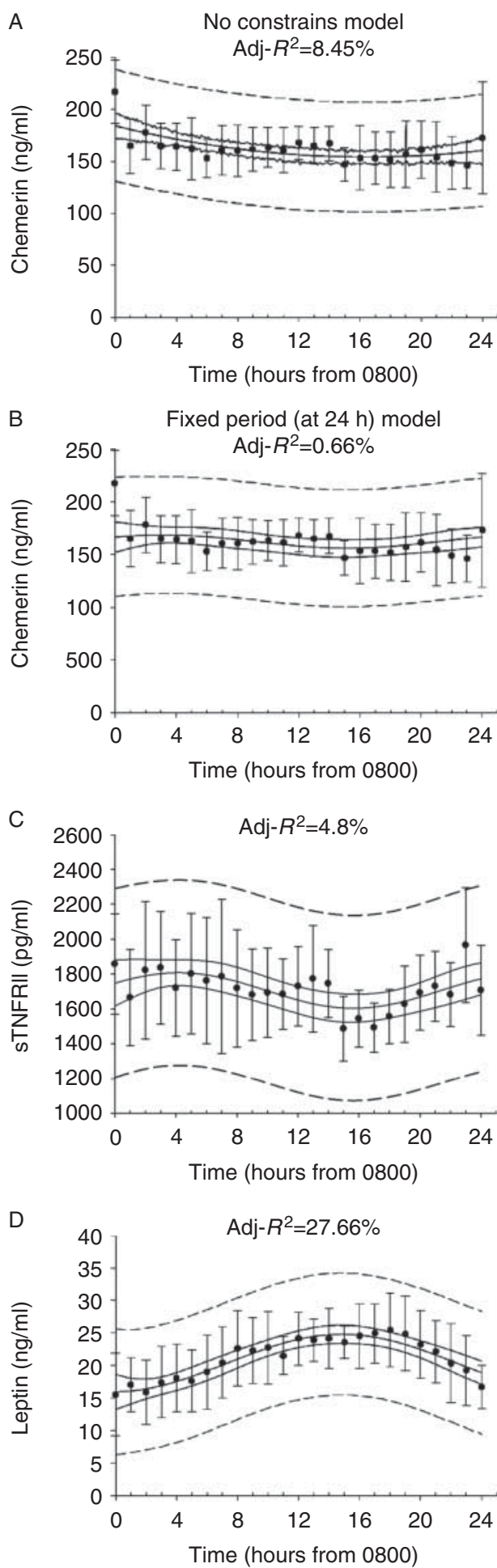

Figure 2 Day/night variation of chemerin. Average 24-h (A) chemerin ( $\mathrm{ng} / \mathrm{ml}$ ) with no constrains, (B) chemerin $(\mathrm{ng} / \mathrm{ml})$ with $24-\mathrm{h}$ fixed period, (C) sTNFRII (pg/ml) and, (D) leptin ( $\mathrm{ng} / \mathrm{ml}$ ) levels (non-linear adjusted $R^{2}$ is displayed on top center of each panel). Error bars represent s.D., continuous line represents $95 \%$ confidence band, and dashed line represents $95 \%$ prediction bands. Adj- $R^{2}$, adjusted coefficient of determination. and the duodenum. Furthermore, it appears that the highest expression of CMKLR1 levels are in tissues involved in inflammatory or immune processes such as the lymph nodes, spleen, and the vena cava. These data suggest that in humans, chemerin might not be an adipocytokine and may rather play a more prominent role in immune/inflammatory response. The latter is consistent with initial studies demonstrating chemerin as a chemoattractant signal for psoriatic skin lesions (2).

Subsequently, we studied the physiology of chemerin in humans, specifically evaluating for any potential day/night variation in chemerin circulating levels and for chemerin's response to starvation and leptin administration in humans. We demonstrate herein, for the first time in humans, that, in contrast to leptin, chemerin does not show a significant day/night oscillation. Although no prior human studies have evaluated this, a 20-h long study on mice with free access to food and water, and blood drawn every $4 \mathrm{~h}$, has previously demonstrated that chemerin exhibits a day/night pattern with a trough exhibited during the night and a peak during the day (4). This is another property of chemerin's physiology that we fail to replicate in humans. The human transcriptome displays a day/night variation (33) and many adipokines, such as leptin and high molecular weight adiponectin, display a diurnal variation in humans $(34,35)$. Interestingly, both the pancreas and liver, which we demonstrate to be two of the prominent sites of chemerin expression, have a vast number of genes, such as the ones encoding proteins essential for glucose metabolism, that express a circadian pattern (36). In our study we demonstrated the absence of any significant day/night oscillation in chemerin circulating levels. We could thus speculate that either chemerin gene expression is not regulated in a circadian fashion in the pancreas and the liver, or that constant, non-circadian secretion from other tissues masks such a pattern. Further studies are encouraged to elucidate this aspect of chemerin's physiology.

We also demonstrate, for the first time in humans, that fasting dramatically affects the levels of circulating chemerin. Fasting for 72-h reduces chemerin levels to $\sim 42 \%$ of that seen during the isocaloric fed state. This suggests that chemerin demonstrates an acute response to changes in energy homeostasis such as acute caloric restriction. Interestingly, chemerin is highly expressed in tissues that are either direct nutrient sensors, such as the pancreas, or in tissues important for relaying nutrient availability such as the adrenal gland, which produces catecholamines during energy deprivation, and the liver. Therefore, chemerin might be another signal of nutrient status, directed toward the parts of the immune tissues that are expressing the highest CMKLR1, indicating prolonged energy deprivation is occurring and therefore decreased activity or energy expenditure from those tissues is required. The latter, however, is only a reasonable speculation derived from 

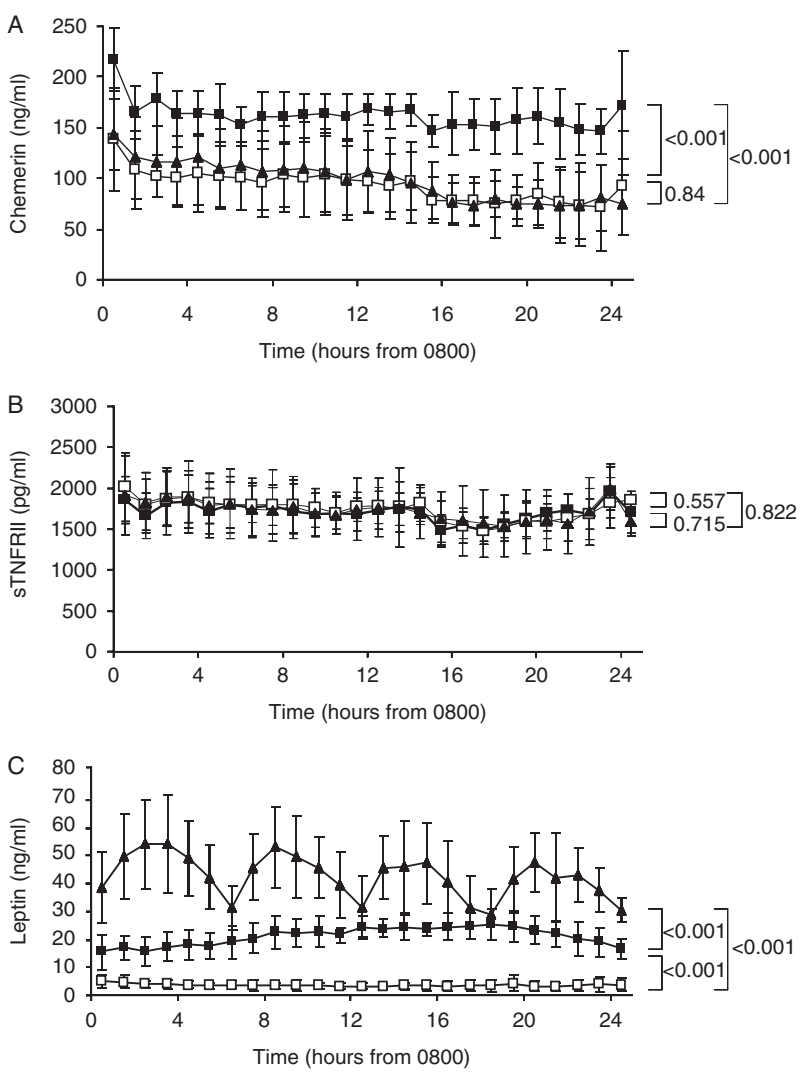

Figure 3 Effects of acute energy deprivation. Average 24-h (A) chemerin ( $\mathrm{ng} / \mathrm{ml}),(B)$ sTNFRII $(\mathrm{pg} / \mathrm{ml})$, and (C) leptin $(\mathrm{ng} / \mathrm{ml})$ levels of fed state (filled square), fasting with placebo administration (open square), and fasting with metreleptin administration (filled triangle). $P$ values are shown next to brackets.

chemerin's expression patterns as well as its response to acute energy deprivation; further translational studies are needed to confirm this. Although sTNFRII levels did not change with fasting, this does not exclude chemerin-induced immune modification in a TNF- $\alpha$ independent fashion.

Chemerin's response to caloric restriction is similar to that of other hormones important in energy homeostasis, such as leptin (35). Leptin is a key molecule signaling information on the amount of energy stored in the adipose tissue and has been shown to be a regulator of metabolic and neuroendocrine adjustment to starvation $(20,35)$. Previous studies from our group have demonstrated that the decrease in leptin levels observed during acute energy deprivation is causally linked to the decrease of other hormones such as luteinizing hormone $(18,35)$, while replacement of leptin to physiologic levels corrects starvation-induced changes of neuroendocrine systems $(35,37)$. Cross-sectional studies have also demonstrated a statistically significant correlation between circulating chemerin and leptin levels in lean, overweight, and type- 2 diabetic humans (12). No study to date has evaluated for any causal relationship between leptin and chemerin. Given the fact that in our study fasting had the same effect on the direction of change in both chemerin and leptin circulating levels we wanted to further evaluate for potential regulation of chemerin circulating levels by leptin. We thus performed an interventional cross-over study of metreleptin and placebo administration in the fasting state on the same subjects whom we used to study for potential day/night variability of chemerin's secretion. We demonstrated that metreleptin replacement has no effect on chemerin levels, implying the lack of any causal relationship between leptin and chemerin. This was also confirmed by our escalating metreleptin dose experiment where administration of low, physiological, and supraphysiological doses of metreleptin during the fed state failed to elicit any dose-response relationship between administered metreleptin dose and measured circulating chemerin levels. Furthermore, we analyzed the effect of $100 \mathrm{ng} / \mathrm{ml}$ of leptin treatment on subcutaneous and omental adipose tissue explants. Chemerin levels in the supernatant of both subcutaneous and omental fat tissue as well as its protein expression levels in the subcutaneous adipose tissue were unaltered after overnight treatment with leptin. We thus can report with confidence that leptin does not affect directly or indirectly circulating chemerin levels or expression, suggesting that a mechanism other than leptin controls the acute response of chemerin to decreased energy intake.

Insulin is another hormone that has been suggested to affect chemerin levels in ex vivo human studies. Specifically, prolonged insulin infusions increase chemerin secretion in human adipose tissue explants (32). Although the drop in chemerin levels observed during
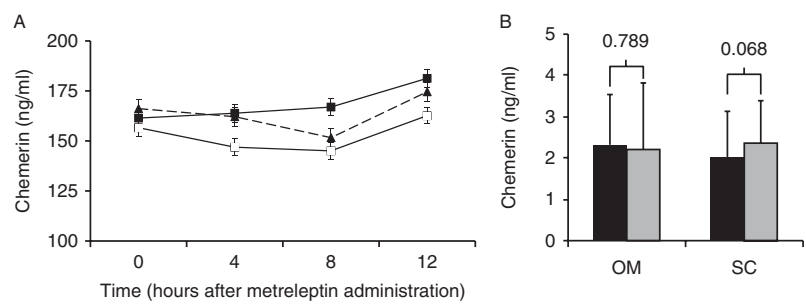

C
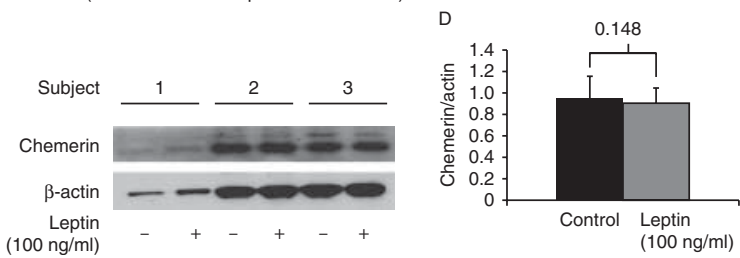

Figure 4 Leptin effects on chemerin. Average 16-h (A) chemerin $(\mathrm{ng} / \mathrm{ml})$ levels after treatment with three doses of leptin (0.01, filled square; 0.1 , open square; and 0.3 , filled triangle, $\mathrm{mg} / \mathrm{kg}$ ). Chemerin levels in supernatant (B) with (gray filled bar) or without (filled bar) $100 \mathrm{ng} / \mathrm{ml}$ leptin treatment for $20 \mathrm{~h}$ in subcutaneous and omental adipose tissue. Chemerin protein expression (C and $D)$ in subcutaneous adipose tissue after leptin treatment. (B, C, and D) Results are the mean of three experiments. Data were analyzed by paired $t$-test. Values are means \pm s.D. 
the fasting state could be theoretically explained by the expected drop in insulin levels, failure of chemerin levels to increase with intake of meals, when insulin levels are expected to increase, surrogates against insulin secretion regulating chemerin levels in vivo. In addition, in our study, chemerin and insulin levels were not correlated in the fasting state at baseline or at day 4 , and there was no significant association in regression of the change of insulin levels between fed and fasting, on chemerin levels. Future studies using long-term insulin administration, possibly through hyperinsulinemic euglycemic clamps, are needed to definitively rule in or out an effect of dropping insulin levels on chemerin during energy deprivation.

Proinflammatory cytokines such as TNF- $\alpha$ have been demonstrated to induce chemerin production in vitro and in vivo $(4,5)$. Production of TNF- $\alpha$ by peripheral blood mononuclear cells increases during acute energy deprivation, while spontaneous TNF- $\alpha$ production has been noted in patients with anorexia nervosa which is a model disease of chronic energy deprivation $(38,39)$. We thus wanted to evaluate whether the decrease in circulating chemerin observed in our study during acute energy deprivation could be attributed to a starvation-induced elevation of circulating TNF- $\alpha$ levels. We thus measured sTNFRII, a reliable marker of TNF- $\alpha$ system activity $(40,41)$, in the subjects undergoing acute energy deprivation. Although we did not measure TNF- $\alpha$ directly, it has been demonstrated that sTNFRII is a more easily and readily detected marker of activation of the TNF- $\alpha$ system that is highly correlated with TNF- $\alpha$ levels (42). Levels of sTNFRII were unaltered by a 72-h fast, indicating that the TNF system is not likely to be responsible for chemerin's changes during short-term energy deprivation.

Our study has several strengths: this is the first study on humans, examining tissue expression of chemerin and its receptor. It is also the first study on humans to evaluate for day/night variability patterns of chemerin secretion and its association with short-term changes in energy homeostasis. The temporal resolution of our sampling intervals is high, enabling us to infer the absence of any potential day/night variation patterns with great confidence. In addition, methods were fully standardized and the subjects were seen at the same period of their menstrual cycle, received the same isocaloric diet, were instructed to keep their exercise levels stable, and were exposed to the same light/dark intervals throughout the study. Furthermore, the randomized cross-over design of this study enables us to control for potential confounding. The $\geq 8$ week interval between study visits enabled adequate wash out of the medication as well as restoration of the subject's hematocrit. The 4-day stay in the GCRC enabled proper standardization of the study conditions. And finally, the use of multiple study groups as well as the data from ex vivo experiments using tissue explants strongly confirms the findings.
One limitation of this study is the small sample size $(n=11)$ of the physiology studies. However, we overcame this limitation by the frequent sampling approach and the cross-over design of the study, which increases study power. This or similar study samples have been proven to be adequate to detect leptin day/night variation patterns, and leptin replacement was able to restore hormonal levels that the 4-day fasting had induced as previously described (25). Also, leptin served as a positive control in this study by demonstrating significant day/night rhythm as well as significant effects due to fasting. Thus, the lack of statistically significant changes in the chemerin day \night variation could not be attributed to the lack of power. Similarly, we confirmed some important findings with other experiments including ex vivo adipose tissue leptin treatment. Furthermore, this study is limited by the use of only female subjects for the analysis of day/night variation and effect of acute energy deprivation, as well as a dosage response to leptin. Future studies must be carried out in a male population to confirm our results are not limited by gender.

In conclusion, our findings show, for the first time, that chemerin has a different tissue-specific expression pattern compared with rodents, and that it is distributed throughout several human tissues, with higher expression in pancreas and liver, suggesting that it may not be functioning as an adipocytokine in humans. Its receptor, CMKLR1, displays the greatest expression in immune tissues such as the spleen and lymph nodes. Furthermore, we demonstrated that, contrary to rodents, chemerin does not exhibit a day/night variation in humans, but is responsive to acute changes in energy homeostasis. We thus recommend clinical studies investigating human circulating chemerin should consider the fasting state of subjects but not necessarily the time of day during blood draws. We also demonstrated that the decreasing levels of chemerin due to starvation are not mediated through leptin or the TNF- $\alpha$ system. These data contribute toward elucidation of chemerin biology in humans and suggest that chemerin physiology in humans is different from rodents and extreme caution should be applied to any attempt to translate findings regarding chemerin from rodent models to human physiology and pathophysiology. Further studies are needed to elucidate the role of chemerin and its regulation in humans.

\section{Declaration of interest}

The authors declare that there is no conflict of interest that could be perceived as prejudicing the impartiality of the research reported.

\section{Funding}

The project described was supported by grant number UL1 RR025758 - Harvard Clinical and Translational Science Center, from the National Center for Research Resources. The content is solely the responsibility of the authors and does not necessarily represent 
the official views of the National Center for Research Resources or the National Institutes of Health. The Mantzoros Laboratory is supported by the National Institute of Diabetes and Digestive and Kidney Diseases Grants 58785, 79929, and 81913 as well as Award Number 1I01CX000422-01A1 from the Clinical Science Research and Development Service of the VA Office of Research and Development. Amylin Pharmaceuticals. Inc. supplied metreleptin for this study but had no role in the study design, conduct of the study, collection, management, analysis, and interpretation of the data, or the preparation, review, or approval of the manuscript.

\section{References}

1 Meder W, Wendland M, Busmann A, Kutzleb C, Spodsberg N, John H, Richter R, Schleuder D, Meyer M \& Forssmann WG. Characterization of human circulating TIG2 as a ligand for the orphan receptor ChemR23. FEBS Letters $2003 \mathbf{5 5 5} 495-499$. (doi:10.1016/S0014-5793(03)01312-7)

2 Nagpal S, Patel S, Jacobe H, DiSepio D, Ghosn C, Malhotra M, Teng M, Duvic M \& Chandraratna RA. Tazarotene-induced gene 2 (TIG2), a novel retinoid-responsive gene in skin. Journal of Investigative Dermatology 1997109 91-95. (doi:10.1111/15231747.ep12276660)

3 Ernst MC \& Sinal CJ. Chemerin: at the crossroads of inflammation and obesity. Trends in Endocrinology and Metabolism 201021 660-667. (doi:10.1016/j.tem.2010.08.001)

4 Parlee SD, Ernst MC, Muruganandan S, Sinal CJ \& Goralski KB. Serum chemerin levels vary with time of day and are modified by obesity and tumor necrosis factor-a. Endocrinology 2010151 2590-2602. (doi:10.1210/en.2009-0794)

5 Kralisch S, Weise S, Sommer G, Lipfert J, Lossner U, Bluher M, Stumvoll M \& Fasshauer M. Interleukin-1 $\beta$ induces the novel adipokine chemerin in adipocytes in vitro. Regulatory Peptides 2009 154 102-106. (doi:10.1016/j.regpep.2009.02.010)

6 Bozaoglu K, Curran JE, Stocker CJ, Zaibi MS, Segal D, Konstantopoulos N, Morrison S, Carless M, Dyer TD, Cole SA et al. Chemerin, a novel adipokine in the regulation of angiogenesis. Journal of Clinical Endocrinology and Metabolism 201095 2476-2485. (doi:10.1210/jc.2010-0042)

7 Kaur J. Adya R, Tan BK. Chen J \& Randeva HS. Identification of chemerin receptor (ChemR23) in human endothelial cells: chemerin-induced endothelial angiogenesis. Biochemical and Biophysical Research Communications 2010391 1762-1768. (doi:10.1016/j.bbrc.2009.12.150)

8 Goralski KB, McCarthy TC, Hanniman EA, Zabel BA, Butcher EC, Parlee SD, Muruganandan S \& Sinal CJ. Chemerin, a novel adipokine that regulates adipogenesis and adipocyte metabolism. Journal of Biological Chemistry 2007282 28175-28188. (doi:10.1074/jbc.M700793200)

9 Roh SG, Song SH, Choi KC, Katoh K, Wittamer V, Parmentier M \& Sasaki S. Chemerin - a new adipokine that modulates adipogenesis via its own receptor. Biochemical and Biophysical Research Communications 2007362 1013-1018. (doi:10.1016/j.bbrc. 2007.08.104)

10 Bozaoglu K, Bolton K, McMillan J, Zimmet P, Jowett J, Collier G, Walder $\mathrm{K}$ \& Segal D. Chemerin is a novel adipokine associated with obesity and metabolic syndrome. Endocrinology $2007 \mathbf{1 4 8}$ 4687-4694. (doi:10.1210/en.2007-0175)

11 Bozaoglu K, Segal D, Shields KA, Cummings N, Curran JE, Comuzzie AG, Mahaney MC, Rainwater DL, VandeBerg JL, MacCluer JW et al. Chemerin is associated with metabolic syndrome phenotypes in a Mexican-American population. Journal of Clinical Endocrinology and Metabolism 200994 3085-3088. (doi:10.1210/jc.2008-1833)

12 Weigert J, Neumeier M, Wanninger J, Filarsky M, Bauer S, Wiest R, Farkas S, Scherer MN, Schaffler A, Aslanidis C et al. Systemic chemerin is related to inflammation rather than obesity in type 2 diabetes. Clinical Endocrinology 201072 342-348. (doi:10.1111/ j.1365-2265.2009.03664.x)
13 Sell H, Divoux A, Poitou C, Basdevant A, Bouillot JL, Bedossa P, Tordjman J, Eckel J \& Clement K. Chemerin correlates with markers for fatty liver in morbidly obese patients and strongly decreases after weight loss induced by bariatric surgery. Journal of Clinical Endocrinology and Metabolism 201095 2892-2896. (doi:10.1210/jc.2009-2374)

14 Tonjes A, Fasshauer M, Kratzsch J, Stumvoll M \& Bluher M. Adipokine pattern in subjects with impaired fasting glucose and impaired glucose tolerance in comparison to normal glucose tolerance and diabetes. PLoS ONE 20105 e13911. (doi:10.1371/ journal.pone.0013911)

15 Takahashi M, Takahashi Y, Takahashi K, Zolotaryov FN, Hong KS, Kitazawa R, Iida K, Okimura Y, Kaji H, Kitazawa S et al. Chemerin enhances insulin signaling and potentiates insulin-stimulated glucose uptake in 3T3-L1 adipocytes. FEBS Letters $2008 \mathbf{5 8 2}$ 573-578. (doi:10.1016/j.febslet.2008.01.023)

16 Zhang Y, Proenca R, Maffei M, Barone M, Leopold L \& Friedman JM. Positional cloning of the mouse obese gene and its human homologue. Nature 1994372 425-432. (doi:10.1038/ $372425 \mathrm{a} 0)$

17 Kelesidis T, Kelesidis I, Chou S \& Mantzoros CS. Narrative review: the role of leptin in human physiology: emerging clinical applications. Annals of Internal Medicine 2010152 93-100. (doi:10.7326/0003-4819-152-2-201001190-00008)

18 Mantzoros CS. Role of leptin in reproduction. Annals of the New York Academy of Sciences 2000900 174-183. (doi:10.1111/ j.1749-6632.2000.tb06228.x)

19 Hamnvik OP, Liu X, Petrou M, Gong H, Chamberland JP, Kim EH, Christophi CA, Kales SN, Christiani DC \& Mantzoros CS. Soluble leptin receptor and leptin are associated with baseline adiposity and metabolic risk factors, and predict adiposity, metabolic syndrome, and glucose levels at 2-year follow-up: the Cyprus Metabolism Prospective Cohort Study. Metabolism 201160 987-993. (doi:10.1016/j.metabol.2010.09.009)

20 Chan JL \& Mantzoros CS. Role of leptin in energy-deprivation states: normal human physiology and clinical implications for hypothalamic amenorrhoea and anorexia nervosa. Lancet 2005 366 74-85. (doi:10.1016/S0140-6736(05)66830-4)

21 Hill JW, Elias CF, Fukuda M, Williams KW, Berglund ED, Holland WL, Cho YR, Chuang JC, Xu Y, Choi M et al. Direct insulin and leptin action on pro-opiomelanocortin neurons is required for normal glucose homeostasis and fertility. Cell Metabolism 201011 286-297. (doi:10.1016/j.cmet.2010.03. 002)

22 Welt CK, Chan JL, Bullen J, Murphy R, Smith P, DePaoli AM, Karalis A \& Mantzoros CS. Recombinant human leptin in women with hypothalamic amenorrhea. New England Journal of Medicine 2004351 987-997. (doi:10.1056/NEJMoa040388)

23 Chou SH, Chamberland JP, Liu X, Matarese G, Gao C, Stefanakis R, Brinkoetter MT, Gong H, Arampatzi K \& Mantzoros CS. Leptin is an effective treatment for hypothalamic amenorrhea. PNAS 2011 108 6585-6590. (doi:10.1073/pnas.1015674108)

24 Sienkiewicz E, Magkos F, Aronis KN, Brinkoetter M, Chamberland JP, Chou S, Arampatzi KM, Gao C, Koniaris A \& Mantzoros CS. Long-term metreleptin treatment increases bone mineral density and content at the lumbar spine of lean hypoleptinemic women. Metabolism: Clinical and Experimental 201160 1211-1221. (doi:10.1016/j.metabol.2011.05.016)

25 Chan JL, Matarese G, Shetty GK, Raciti P, Kelesidis I, Aufiero D, De Rosa V, Perna F, Fontana S \& Mantzoros CS. Differential regulation of metabolic, neuroendocrine, and immune function by leptin in humans. PNAS 2006103 8481-8486. (doi:10.1073/ pnas.0505429103)

26 Chan JL, Wong SL \& Mantzoros CS. Pharmacokinetics of subcutaneous recombinant methionyl human leptin administration in healthy subjects in the fed and fasting states: regulation by gender and adiposity. Clinical Pharmacokinetics 200847 753-764. (doi:10.2165/00003088-200847110-00006)

27 Moon HS, Chamberland JP, Diakopoulos KN, Fiorenza CG, Ziemke F, Schneider B \& Mantzoros CS. Leptin and amylin act 
in an additive manner to activate overlapping signaling pathways in peripheral tissues: in vitro and ex vivo studies in humans. Diabetes Care 201034 132-138. (doi:10.2337/dc10-0518)

28 Chakaroun R, Raschpichler M, Kloting N, Oberbach A, Flehmig G, Kern M, Schon MR, Shang E, Lohmann T, Dressler M et al. Effects of weight loss and exercise on chemerin serum concentrations and adipose tissue expression in human obesity. Metabolism: Clinical and Experimental $2012 \mathbf{6 1}$ 706-714. (doi:10.1016/j.metabol. 2011.10.008)

29 El-Mesallamy HO, El-Derany MO \& Hamdy NM. Serum omentin-1 and chemerin levels are interrelated in patients with type 2 diabetes mellitus with or without ischaemic heart disease. Diabetic Medicine 201128 1194-1200. (doi:10.1111/j.14645491.2011.03353.x)

30 Vamvini MT, Aronis KN, Chamberland JP \& Mantzoros CS. Energy deprivation alters in a leptin- and cortisol-independent manner circulating levels of activin A and follistatin but not myostatin in healthy males. Journal of Clinical Endocrinology and Metabolism 201196 3416-3423. (doi:10.1210/jc.2011-1665)

31 Wittamer V, Franssen JD, Vulcano M, Mirjolet JF, Le Poul E, Migeotte I, Brezillon S, Tyldesley R, Blanpain C, Detheux M et al. Specific recruitment of antigen-presenting cells by chemerin, a novel processed ligand from human inflammatory fluids. Journal of Experimental Medicine 2003198 977-985. (doi:10.1084/jem. 20030382)

32 Tan BK, Chen J, Farhatullah S, Adya R, Kaur J, Heutling D, Lewandowski KC, O'Hare JP, Lehnert H \& Randeva HS. Insulin and metformin regulate circulating and adipose tissue chemerin. Diabetes 200958 1971-1977. (doi:10.2337/db08-1528)

33 Loboda A, Kraft WK, Fine B, Joseph J, Nebozhyn M, Zhang C, He Y, Yang X, Wright C, Morris M et al. Diurnal variation of the human adipose transcriptome and the link to metabolic disease. BMC Medical Genomics 20092 7. (doi:10.1186/1755-8794-2-7)

34 Scheer FA, Chan JL, Fargnoli J, Chamberland J, Arampatzi K, Shea SA, Blackburn GL \& Mantzoros CS. Day/night variations of high-molecular-weight adiponectin and lipocalin-2 in healthy men studied under fed and fasted conditions. Diabetologia 201053 2401-2405. (doi:10.1007/s00125-010-1869-7)
35 Mullington JM, Chan JL, Van Dongen HP, Szuba MP, Samaras J, Price NJ, Meier-Ewert HK, Dinges DF \& Mantzoros CS. Sleep loss reduces diurnal rhythm amplitude of leptin in healthy men. Journal of Neuroendocrinology 200315 851-854. (doi:10.1046/ j.1365-2826.2003.01069.x)

36 Kalsbeek A, Ruiter M, La Fleur SE, Cailotto C, Kreier F \& Buijs RM. The hypothalamic clock and its control of glucose homeostasis. Progress in Brain Research 2006153 283-307.

37 Mantzoros CS, Cramer DW, Liberman RF \& Barbieri RL. Predictive value of serum and follicular fluid leptin concentrations during assisted reproductive cycles in normal women and in women with the polycystic ovarian syndrome. Human Reproduction 200015 539-544. (doi:10.1093/humrep/15.3.539)

38 Vaisman N, Schattner A \& Hahn T. Tumor necrosis factor production during starvation. American Journal of Medicine 1989 87 115. (doi:10.1016/S0002-9343(89)80497-8)

39 Vaisman N \& Hahn T. Tumor necrosis factor- $\alpha$ and anorexia cause or effect? Metabolism 199140 720-723. (doi:10.1016/ 0026-0495(91)90090-J)

40 Diez-Ruiz A, Tilz GP, Zangerle R, Baier-Bitterlich G, Wachter H \& Fuchs D. Soluble receptors for tumour necrosis factor in clinical laboratory diagnosis. European Journal of Haematology $1995 \mathbf{5 4}$ 1-8. (doi:10.1111/j.1600-0609.1995.tb01618.x)

41 Mantzoros CS, Moschos S, Avramopoulos I, Kaklamani V, Liolios A, Doulgerakis DE, Griveas I, Katsilambros N \& Flier JS. Leptin concentrations in relation to body mass index and the tumor necrosis factor- $\alpha$ system in humans. Journal of Clinical Endocrinology and Metabolism 199782 3408-3413. (doi:10.1210/jc.82.10.3408)

42 Aderka D. The potential biological and clinical significance of the soluble tumor necrosis factor receptors. Cytokine $\&$ Growth Factor Reviews 19967 231-240. (doi:10.1016/S1359-6101(96) 00026-3)

Received 31 January 2013

Revised version received 21 July 2013

Accepted 31 July 2013 